Louisiana State University

LSU Digital Commons

Faculty Publications

Department of Mathematics

$4-1-2020$

\title{
Continuous Discrete Sequential Observers for Time-Varying Systems under Sampling and Input Delays
}

Frederic Mazenc

Laboratoire des Signaux et Systèmes

Michael Malisoff

Louisiana State University

Follow this and additional works at: https://digitalcommons.Isu.edu/mathematics_pubs

\section{Recommended Citation}

Mazenc, F., \& Malisoff, M. (2020). Continuous Discrete Sequential Observers for Time-Varying Systems under Sampling and Input Delays. IEEE Transactions on Automatic Control, 65 (4), 1704-1709.

https://doi.org/10.1109/TAC.2019.2930676

This Article is brought to you for free and open access by the Department of Mathematics at LSU Digital Commons. It has been accepted for inclusion in Faculty Publications by an authorized administrator of LSU Digital Commons. For more information, please contact ir@lsu.edu. 
archives-ouvertes

\section{Continuous-Discrete Sequential Observers for Time-Varying Systems under Sampling and Input Delays}

Frédéric Mazenc, Michael Malisoff

\section{To cite this version:}

Frédéric Mazenc, Michael Malisoff. Continuous-Discrete Sequential Observers for Time-Varying Systems under Sampling and Input Delays. IEEE Transactions on Automatic Control, Institute of Electrical and Electronics Engineers, 2019, 10.1109/TAC.2019.2930676 . hal-02342849

HAL Id: hal-02342849

https://hal.inria.fr/hal-02342849

Submitted on 1 Nov 2019

HAL is a multi-disciplinary open access archive for the deposit and dissemination of scientific research documents, whether they are published or not. The documents may come from teaching and research institutions in France or abroad, or from public or private research centers.
L'archive ouverte pluridisciplinaire HAL, est destinée au dépôt et à la diffusion de documents scientifiques de niveau recherche, publiés ou non, émanant des établissements d'enseignement et de recherche français ou étrangers, des laboratoires publics ou privés. 


\title{
Continuous-Discrete Sequential Observers for
}

\section{Time-Varying Systems under Sampling and Input Delays}

\author{
Frederic Mazenc and Michael Malisoff, Senior Member, IEEE
}

\begin{abstract}
We provide continuous-discrete observers for a large class of time-varying linear systems where the inputs and outputs have sampling and delays, and where the systems and outputs contain uncertainties. We allow the delays in the output and input to differ and to be arbitrarily long. We use the observers to design controls that ensure input-to-state stability, under delays and sampling. The observers and controls have no distributed terms. We illustrate our work in two examples including a DC motor model.
\end{abstract}

\section{INTRODUCTION}

Global asymptotic stabilization of systems with delays or outputs can be challenging when the output is not available for continuous measurement. This has motivated a large literature on stabilization problems under discrete sampling of an output, which have important engineering implications [1]. Notable tools in this literature include prediction and reduction model approaches (and other methods) for delay compensation, which can be advantageous in applications because they can compensate for arbitrarily long input delays. Prediction and reduction model approaches construct dynamic extensions that generally lead to distributed terms in controls, so the control formulas often incorporate an integral of past control or state values.

Reduction model methods in [2] were confined to cases with continuous measurements of the complete state, but [2] covered linear systems with piecewise constant inputs. For systems with only discrete output measurements, several methods exist. For example, if the input delay is a multiple of the constant length $\bar{c}$ of all sampling time intervals in the input and output measurements, then one can apply a discrete time reduction model method from [3]; see [4].

Index Terms: Delays, observers, sampling. Supported by US National Science Foundation Grant 1711299. Mazenc is with EPI DISCO INRIA-Saclay, Laboratoire des Signaux et Systèmes (L2S, UMR CNRS 8506), CNRS, CentraleSupélec, Université Paris-Sud, 3 rue Joliot Curie, 91192, Gif-sur-Yvette, France, frederic.mazenc@12s.centralesupelec.fr. Malisoff is with the Department of Mathematics, Louisiana State University, Baton Rouge, LA 70803-4918, USA, malisoff@1su.edu. 
The seminal works [5] and [6] and more recent works (e.g., [2], [7]) used continuous-discrete observers, which apply in cases that are not amenable to static piecewise constant state feedback or to continuous observers. The work [8] used continuous-discrete observers to build globally asymptotically stabilizing output feedback under input delays, and [8] combined reduction model methods with continuous-discrete observers. Other works treated delays, discrete measurements, and sampling separately (but see the nonlinear stabilization work [9] for systems with inputs with zero-order hold under discrete delayed inputs). By contrast, here we use the sequential observers and subsequential predictors approach that was started in [10] and that was further developed in [11], and that eliminates the need for distributed terms in the control. For the first time, this paper adapts this approach to cases where continuous-discrete observers are appropriate, and we allow time-varying systems with different delays in the input and output, under sampling.

The work [8] for LTI systems with sampling in the input and the output uses a dynamic extension. However, the distributed terms in works like [8] and [9] may not always lend themselves to implementations, since the controls are not in a closed form. In the present work, our closed form stabilizing controls apply to linear time-varying systems with sampling in the input and in the output, and uncertainties in the output measurements and in the original plant. Then we can prove input-to-state stability under arbitrarily long delays in the input and output.

This work adds significant value relative to our conference version [12] by (i) covering linear time-varying systems (which arise from tracking reference trajectories), (ii) providing sufficient conditions for our Schur matrix condition to hold, and (iii) including an application to a DC motor model. These three features were not present in [12], which was confined to LTI systems.

\section{Statement of Main Result}

We study systems having the form

$$
\left\{\begin{array}{l}
\dot{x}(t)=A(t) x(t)+B(t) u(g(t)-\tau)+\delta_{1}(t) \\
y\left(t_{i}\right)=C\left(t_{i}\right) x\left(t_{i-r}\right)+\delta_{2}\left(t_{i}\right), \quad i \geq r
\end{array}\right.
$$

where $t_{i}=i \nu$ for a known constant $\nu>0$ for all integers $i \geq 0$, the constant input and output delays $\tau \geq 0$ and $r \in \mathbb{N}$ and the bounded continuous matrix valued functions $A, B$, and $C$ are known, the state $x$ and the output $y$ are valued in $\mathbb{R}^{n_{x}}$ and $\mathbb{R}^{n_{y}}$ respectively for any dimensions $n_{x}$ and $n_{y}, g$ is a right continuous nondecreasing piecewise continuous unbounded function that satisfies $g(t) \leq t$ for all $t \geq 0$ and so can represent sampling in the control $u$ (e.g., by choosing $g(t)=s_{i}$ for all $t \in\left[s_{i}, s_{i+1}\right)$, where $s_{0}=0$ and where the sample times $s_{i}$ admit 
positive constants $\underline{s}$ and $\bar{s}$ such that $\underline{s} \leq s_{i+1}-s_{i} \leq \bar{s}$ for all integers $i \geq 0$ ), and the unknown $\delta=\left(\delta_{1}, \delta_{2}\right)$ is piecewise continuous and bounded and has finite right limits at each $t \geq 0$. Including $g(t)$ is motivated by sampling that commonly occurs when controls are implemented, and by the challenge of proving robustness to sampling [8]. We use standard definitions for the input-to-state stable (or ISS) property; see [13]. We assume that our initial functions for (1) are constant at the initial time $t_{0}=0, I$ denotes an identity matrix, and $\Phi_{\mathcal{C}}$ is the fundamental matrix for $\dot{z}(t)=\mathcal{C}(t) z(t)$ for any continuous choice of $\mathcal{C}$. We assume the following, where $M_{a} \leq M_{b}$ for square matrices $M_{a}$ and $M_{b}$ of the same size means $M_{b}-M_{a}$ is nonnegative definite, $|\cdot|$ is the usual Euclidean norm and the induced matrix norm, and $|\cdot|_{\infty}$ is the essential supremum:

Assumption 1. There are constant matrices $A_{0} \in \mathbb{R}^{n_{x} \times n_{x}}, C_{0} \in \mathbb{R}^{n_{y} \times n_{x}}$, and $L_{0} \in \mathbb{R}^{n_{x} \times n_{y}}$; an integer $d$ and a constant $\ell>1$ such that $d=\ell \tau / \nu$; bounded piecewise continuous functions $\delta_{A}: \mathbb{R} \rightarrow \mathbb{R}^{n_{x} \times n_{x}}$ and $\delta_{C}: \mathbb{R} \rightarrow \mathbb{R}^{n_{y} \times n_{x}} ;$ and a symmetric positive definite $P_{0} \in \mathbb{R}^{n_{x} \times n_{x}}$ such that:

(i) the equalities $A(t)=A_{0}+\delta_{A}(t)$ and $C(t)=C_{0}+\delta_{C}(t)$ hold for all $t \in \mathbb{R}$;

(ii) the matrix $M_{0}(\nu)=\left(I+\nu L_{0} C_{0} e^{-(\tau / d) A_{0}}\right) e^{\nu A_{0}}$ is such that $M_{0}^{\top}(\nu) P_{0} M_{0}(\nu)-P_{0} \leq-I$; and

(iii) the pair $\delta_{*}=\left(\delta_{A}, \delta_{C}\right)$ is such that $\left|P_{0}\right| \mathcal{G}\left(\delta_{*}\right) \mathcal{H}\left(\delta_{*}\right)<1$, where $\mathcal{G}\left(\delta_{*}\right)=e^{\nu\left|A_{0}\right|}\left(e^{\nu\left|\delta_{A}\right|_{\infty}}-\right.$ $1)\left(1+\nu\left|L_{0}\right||C|_{\infty}\right)+\nu\left|L_{0}\right|\left|\delta_{C}\right|_{\infty} e^{\nu\left|A_{0}\right|}$ and $\mathcal{H}\left(\delta_{*}\right)=\left(1+\nu\left|L_{0} C\right|_{\infty}\right) e^{\nu|A|_{\infty}}+\left|M_{0}(\nu)\right|$.

When $\delta_{A}$ and $\delta_{C}$ are zero functions, we can find a $P_{0}$ to satisfy part (ii) of Assumption 1 if $(I+L C) e^{\nu A}$ is Schur stable, by choosing $L=\nu L_{0}$ (and choosing $d$ large enough and using the continuity of eigenvalues as functions of the entries of the matrix). If $\left(A_{0}, C_{0}\right)$ is observable, then Appendix A.1 below shows a way to satisfy condition (ii) of Assumption 1. Since the Mean Value Theorem gives $e^{\nu\left|\delta_{A}\right|_{\infty}}-1 \leq e^{\nu\left|\delta_{A}\right|_{\infty}} \nu\left|\delta_{A}\right|_{\infty}$, condition (iii) from Assumption 1 then holds if $\nu\left|\delta_{*}\right|_{\infty}$ is sufficiently small. For instance, if $\nu \in(0,1)$ and $\left|\delta_{*}\right|_{\infty} \leq 1$, then $\mathcal{G}\left(\delta_{*}\right) \leq \overline{\mathcal{G}}\left|\delta_{*}\right|_{\infty} \nu$ and $\mathcal{H}\left(\delta_{*}\right) \leq \overline{\mathcal{H}}$, where $\overline{\mathcal{G}}=e^{\left|A_{0}\right|+1}\left[1+\left|L_{0}\right|\left(\left|C_{0}\right|+1\right)\right]+\left|L_{0}\right| e^{\left|A_{0}\right|}$ and $\overline{\mathcal{H}}=e^{\left|A_{0}\right|}\left(e\left(1+\left|L_{0}\right|\left(\left|C_{0}\right|+1\right)\right)+1+\left|L_{0} C_{0}\right| e^{\tau\left|A_{0}\right|}\right)$, so part (iii) of Assumption 1 holds if $\nu$ and $\delta_{*}$ also satisfy $\nu\left|\delta_{*}\right|_{\infty}<\frac{1}{\left|P_{0}\right| \mathcal{G H}}$. While [8] allows outputs and nonperiodic sampling, [8] leads to distributed terms in controls, even when $A, B$, and $C$ are constant matrices. Finally, we assume:

Assumption 2. The ratio $\tau / \nu$ is a rational number.

We prove the following, where + refers to the right limit, and $|\cdot|_{\mathcal{I}}$ is the supremum over any interval $\mathcal{I}$; see Remark 1 for the definition of a solution for (2a)-(2b). 
Theorem 1. Let Assumptions 1-2 hold for some positive integer $d>\max \{3, \tau / \nu\}$ and some constant $\ell>1$, and choose $L_{0}$ from Assumption 1. Set $p=d+\ell r$, and consider the families of continuous-discrete systems

$$
\begin{aligned}
& \left\{\begin{aligned}
\dot{z}_{1}(t) & =A\left(\phi_{1}(t)\right) z_{1}(t)+B\left(\phi_{1}(t)\right) u\left(g\left(t+\frac{\tau}{d}-r \nu\right)-\tau\right) \forall t \in J_{i} \\
z_{1}\left(t_{i}^{+}\right) & =z_{1}\left(t_{i}\right)+\nu L_{0}\left[C\left(t_{i}\right) z_{1}\left(t_{i}-\frac{\tau}{d}\right)-y\left(t_{i}\right)\right], i \geq r
\end{aligned}\right. \\
& \text { and }\left\{\begin{aligned}
\dot{z}_{j}(t) & =A\left(\phi_{j}(t)\right) z_{j}(t)+B\left(\phi_{j}(t)\right) u\left(g\left(t+\frac{j \tau}{d}-r \nu\right)-\tau\right) \forall t \in J_{i} \\
z_{j}\left(t_{i}^{+}\right) & =z_{j}\left(t_{i}\right)+\nu L_{0} C\left(t_{i}\right) \psi_{j}\left(t_{i}-\frac{\tau}{d}\right), i \geq r
\end{aligned}\right.
\end{aligned}
$$

for $j=2, \ldots, p$, where $J_{i}=\left(t_{i}, t_{i+1}\right]$ for $i \geq 0$ and we set $\psi_{1}(t)=z_{1}(t)-x\left(\phi_{1}(t)\right)$ and $\psi_{j}(t)=z_{j}(t)-z_{j-1}\left(t+\frac{\tau}{d}\right)$ for $j=2, \ldots, p$ for any choice of the piecewise continuous locally bounded function $u$, and where $\phi_{j}(t)=t+\frac{j \tau}{d}-r \nu$ for all $t \geq 0$ and $j \in\{1,2, \ldots, p\}$, and where $A, B, C, u$, and $g$ are from (1). Set $\Psi=\left(\psi_{1}, \ldots, \psi_{p}\right)$. Then the following conclusions hold: (a) We can find positive constants $c_{1}$ and $c_{2}$ such that $|\Psi(t)| \leq c_{1}\left(e^{-c_{2} t}\left|\Psi\left(t_{r}^{+}\right)\right|+|\delta|_{[0, t]}\right)$ holds for all $t \geq t_{r}$ and for all solutions of (1) and (2a)-(2b). (b) The estimate

$$
\left|z_{p}(t-\tau)-x(t)\right| \leq p c_{1}\left(e^{-c_{2}(t-\tau)}\left|\Psi\left(t_{r}^{+}\right)\right|+|\delta|_{[0, t]}\right)
$$

holds for all $t \geq t_{r}+\tau$ along all solutions of (1) and (2a)-(2b). (c) If, in addition, there is constant matrix $K$ such that $\dot{x}(t)=A(t) x(t)+B(t) K x(g(t))+\delta_{a}(t)$ is exponentially ISS with respect to the arbitrary locally bounded piecewise continuous uncertainty $\delta_{a}$, then the $(x, \Psi)$ dynamics, consisting of (1) and the $\Psi=\left(\psi_{1}, \ldots, \psi_{p}\right)$ system in closed loop with $u(t)=K z_{p}(t)$, are ISS with respect to $\delta=\left(\delta_{1}, \delta_{2}\right)$.

Remark 1. Solutions of (2a)-(2b) are understood as follows. The $z_{j}$ subsystems (called sequential observers) are continuous time ones (whose dynamics are defined by the first equations in (2a)(2b) for all $t>0$ ), except their states are reset to $z_{j}\left(t_{i}^{+}\right)$at the times $t_{i}$. Then $z_{p}$ provides an observer for the state $x$ of (1), by conclusion (b). A guide for finding $K$ in part (c) is to choose $K$ such that $\dot{x}(t)=[A(t)+B(t) K] x(t)$ is uniformly globally exponentially stable to 0 (which is possible, e.g., if $(A(0), B(0))$ is a controllable pair and $K$ is such that $A(0)+B(0) K$ is Hurwitz, under suitable bounds on the time variation of $A$ and $B$, by simple calculations), since then the requirements hold if $\sup _{t \geq 0}|t-g(t)|$ is small enough, by converse Lyapunov function theory (e.g., [13, Theorem 4.14]) and [14], which give an ISS Lyapunov function for $\dot{x}(t)=A(t) x(t)+B(t) K x(g(t))+\delta_{a}(t)$. Our condition $d>\max \{3, \tau / \nu\}$ shows that smaller $\nu$ 's produce higher dimensional observers (but our control and observers are free of distributed terms for all $r$ and $\tau$ ). It is tempting to guess that robustness in [12] can be used to directly 
extend [12] to linear time-varying systems, but this would not be right because the robustness in [12] is to added uncertainties on the right side, not uncertainties in coefficient matrices.

\section{Proof OF TheOREM 1}

We divide the proof into three parts. In the first part, we show that part (ii) of Assumption 1 remains true (up to a scaling of $P_{0}$ by a positive constant) when $M_{0}(\nu)$ in its inequality is replaced by a suitable analog of $M_{0}(\nu)$ for time-varying linear systems. The second part provides key relations that enable us to prove the conclusions (a)-(c) of the theorem in the third part.

First Part. Setting $L=\nu L_{0}$, we prove that there is a constant $c_{0}>0$ such that the matrices $M_{i j}=\Phi_{A \circ \phi_{j}}\left(t_{i}, t_{i-1}\right)+L C\left(t_{i}\right) \Phi_{A \circ \phi_{j}}\left(t_{i}-\tau / d, t_{i-1}\right)$ satisfy $M_{i j}^{\top} P_{0} M_{i j}-P_{0} \leq-c_{0} I$ for all integers $i \geq 0$ and $j \in\{1,2, \ldots, p\}$. We will use the facts (which follow from Appendix A.2 with the choices $\left.\mathcal{A}=A \circ \phi_{j}\right)$ that $\left|\Phi_{A \circ \phi_{j}}(t, s)-e^{A_{0}(t-s)}\right| \leq e^{(t-s)\left|A_{0}\right|}\left(e^{(t-s)\left|\delta_{A}\right|_{\infty}}-1\right)$ and $\left|\Phi_{A \circ \phi_{j}}(t, s)\right| \leq e^{|A|_{\infty}(t-s)}$ hold for all $s \geq 0$ and $t \geq s$ and $j$. By the triangle inequality, for all integers $i \geq 0$ and $j \in\{1,2, \ldots, p\}$, our choice of $\mathcal{G}$ in part (iii) of Assumption 1 is such that

$$
\begin{aligned}
\left|M_{i j}-M_{0}(\nu)\right| \leq & \left|e^{\nu A_{0}}-\Phi_{A \circ \phi_{j}}\left(t_{i}, t_{i-1}\right)\right| \\
& +\left|L\left[C_{0}-C\left(t_{i}\right)\right] e^{(\nu-\tau / d) A_{0}}\right|+\left|L C\left(t_{i}\right)\left(e^{(\nu-\tau / d) A_{0}}-\Phi_{A \circ \phi_{j}}\left(t_{i}-\frac{\tau}{d}, t_{i-1}\right)\right)\right| \\
\leq & e^{\nu\left|A_{0}\right|}\left(e^{\nu\left|\delta_{A}\right|_{\infty}}-1\right)+|L|\left|\delta_{C}\right|_{\infty} e^{\nu\left|A_{0}\right|} \\
& +\nu\left|L_{0}\right|\left|C_{0}+\delta_{C}\right|_{\infty} e^{\nu\left|A_{0}\right|}\left(e^{\nu\left|\delta_{A}\right|_{\infty}}-1\right)=\mathcal{G}\left(\delta_{*}\right)
\end{aligned}
$$

by using the estimates $\left|L \delta_{C}\left(t_{i}\right) e^{(\nu-\tau / d) A_{0}}\right| \leq|L|\left|\delta_{C}\right|_{\infty} e^{|\nu-\tau / d|\left|A_{0}\right|} \leq|L|\left|\delta_{C}\right|_{\infty} e^{\nu\left|A_{0}\right|}$ which follow because $d \geq \tau / \nu$, and recalling the $\nu\left|L_{0}\right|\left|\delta_{C}\right|_{\infty} e^{\nu\left|A_{0}\right|}$ in the $\mathcal{G}\left(\delta_{*}\right)$ formula and the fact that $L=\nu L_{0}$. Also, $\left|M_{i j}\right| \leq e^{\nu|A|_{\infty}}\left(1+|L C|_{\infty}\right)$ and so also $\left|M_{i j}+M_{0}(\nu)\right| \leq \mathcal{H}\left(\delta_{*}\right)$ for all $i$ and $j$. Hence, we can use (iii) from Assumption 1 and the matrix difference of squares formula to find a constant $c_{0} \in(0,1)$ such that $V^{\top}\left(M_{i j}^{\top} P_{0} M_{i j}-P_{0}\right) V=V^{\top}\left(M_{0}^{\top}(\nu) P_{0} M_{0}(\nu)-P_{0}\right) V+$ $V^{\top}\left(M_{i j}^{\top}-M_{0}^{\top}(\nu)\right) P_{0}\left(M_{i j}+M_{0}(\nu)\right) V \leq-c_{0}|V|^{2}$ for all $i \geq 0, V \in \mathbb{R}^{n_{x}}$, and $j=1,2, \ldots, p$.

Second Part. Let $P=P_{0} / c_{0}$, where $c_{0}$ is from the first part of the proof. Then $M_{i j}^{\top} P M_{i j}-P \leq$ $-I$ for all $i \geq 0$ and $j=1,2, \ldots, p$. From the definition of $\left\{t_{i}\right\}$, we can rewrite (2a) as $\dot{z}_{1}(t)=A\left(\phi_{1}(t)\right) z_{1}(t)+B\left(\phi_{1}(t)\right) u\left(g\left(t+\frac{\tau}{d}-r \nu\right)-\tau\right)$ for all $t \in J_{i}$, and $z_{1}\left(t_{i}^{+}\right)=z_{1}\left(t_{i}\right)+$ $L C\left(t_{i}\right)\left[z_{1}\left(t_{i}-\frac{\tau}{d}\right)-x\left(t_{i}-r \nu\right)\right]-L \delta_{2}\left(t_{i}\right)$ for all $i \geq r$, because $L=\nu L_{0}$. Since $\dot{x}\left(\phi_{1}(t)\right)=$ $A\left(\phi_{1}(t)\right) x\left(\phi_{1}(t)\right)+B\left(\phi_{1}(t)\right) u\left(g\left(\phi_{1}(t)\right)-\tau\right)+\delta_{1}\left(\phi_{1}(t)\right)$ for all $t \geq 0$, it follows from the preceding formulas for $\dot{z}_{1}(t)$ and $z_{1}\left(t_{i}^{+}\right)$that $\psi_{1}$ as defined in our theorem satisfies

$$
\left\{\begin{aligned}
\dot{\psi}_{1}(t) & =A\left(\phi_{1}(t)\right) \psi_{1}(t)-\delta_{1}\left(t+\frac{\tau}{d}-r \nu\right), \forall t \in J_{i} \\
\psi_{1}\left(t_{i}^{+}\right) & =z_{1}\left(t_{i}\right)+\nu L_{0} C\left(t_{i}\right)\left[z_{1}\left(t_{i}-\frac{\tau}{d}\right)-x\left(t_{i}-r \nu\right)\right]-x\left(t_{i}+\frac{\tau}{d}-r \nu\right)-\nu L_{0} \delta_{2}\left(t_{i}\right) \\
& =\psi_{1}\left(t_{i}\right)+\nu L_{0} C\left(t_{i}\right) \psi_{1}\left(t_{i}-\frac{\tau}{d}\right)-\nu L_{0} \delta_{2}\left(t_{i}\right)
\end{aligned}\right.
$$


for all $i \geq r$. Since $t_{i}-\frac{\tau}{d} \in\left(t_{i-1}, t_{i}\right)$ for all $i \geq 1$ (because $t_{i+1}-t_{i}=\nu>\tau / d$ for all $i$ ), we can integrate the first equality in (4) along $\left(t_{i-1}, t_{i}\right]$ and then on $\left(t_{i-1}, t_{i}-\tau / d\right]$ using variation of parameters, and then substitute the resulting formulas for $\psi_{1}\left(t_{i}\right)$ and $\psi_{1}\left(t_{i}-\tau / d\right)$ into the last expression for $\psi_{1}\left(t_{i}^{+}\right)$in (4), to obtain

$$
\begin{aligned}
& \psi_{1}\left(t_{i}^{+}\right)=M_{i 1} \psi_{1}\left(t_{i-1}^{+}\right)+\mathcal{N}_{0}(\delta, i), \text { where } \\
& \mathcal{N}_{0}(\delta, i)=-\int_{t_{i-1}}^{t_{i}} \mathcal{M}_{*}\left(\delta, t_{i}, w\right) \mathrm{d} w-\nu L_{0} \delta_{2}\left(t_{i}\right)-\nu L_{0} C\left(t_{i}\right) \int_{t_{i-1}}^{t_{i}-\tau / d} \mathcal{M}_{*}\left(\delta, t_{i}-\tau / d, w\right) \mathrm{d} w
\end{aligned}
$$

and $\mathcal{M}_{*}(\delta, s, w)=\Phi_{A \circ \phi_{1}}(s, w) \delta_{1}(w+\tau / d-r \nu)$. Let $z_{0}(t)=x(t-r \nu)$. Then we have

$$
\begin{aligned}
\dot{z}_{j}(t) & =A\left(\phi_{j}(t)\right) z_{j}(t)+B\left(\phi_{j}(t)\right) u\left(\mathcal{G}_{*}(t, j)-\tau\right) \quad \forall t \in J_{i} \\
z_{j}\left(t_{i}^{+}\right) & =z_{j}\left(t_{i}\right)-\nu L_{0} \Delta_{j}\left(t_{i}\right)+\nu L_{0}\left[C\left(t_{i}\right) z_{j}\left(t_{i}-\frac{\tau}{d}\right)-C\left(t_{i}\right) z_{j-1}\left(t_{i}\right)\right], i \geq r
\end{aligned}
$$

for all $j \in\{1, \ldots, p\}$, where $\mathcal{G}_{*}(t, s)=g\left(t+\frac{s \tau}{d}-r \nu\right)$, and where $\Delta_{k}=\delta_{2}$ if $k=1$ and $\Delta_{k}=0$ otherwise. Consequently, since $\tau / d<\nu$, it follows that for all $j \in\{2, \ldots, p\}$, we have

$$
\dot{\psi}_{j}(t)=A\left(\phi_{j}(t)\right) \psi_{j}(t) \forall t \in J_{i} \text {, and } \psi_{j}\left(t_{i}^{+}\right)=\psi_{j}\left(t_{i}\right)+\nu L_{0} C\left(t_{i}\right) \psi_{j}\left(t_{i}-\tau / d\right) \quad \forall i \geq r .
$$

By integrating the first equation in (7) over $\left(t_{i}-\frac{\tau}{d}, t_{i}\right]$, we obtain $\psi_{j}\left(t_{i}\right)=\Phi_{A \circ \phi_{j}}\left(t_{i}, t_{i}-\right.$ $\left.\frac{\tau}{d}\right)\left[z_{j}\left(t_{i}-\frac{\tau}{d}\right)-z_{j-1}\left(t_{i}^{+}\right)\right]$. By (6), it follows that $\psi_{j}\left(t_{i}\right)=\Phi_{A \circ \phi_{j}}\left(t_{i}, t_{i}-\frac{\tau}{d}\right)\left[z_{j}\left(t_{i}-\frac{\tau}{d}\right)-z_{j-1}\left(t_{i}\right)-\right.$ $\left.\nu L_{0} C\left(t_{i}\right)\left(z_{j-1}\left(t_{i}-\frac{\tau}{d}\right)-z_{j-2}\left(t_{i}\right)\right)+\nu L_{0} \Delta_{j-1}\left(t_{i}\right)\right]$ holds for all $j \in\{2, \ldots, p\}$ and $i \geq r$. Hence, $\psi_{j}\left(t_{i}\right)=\Phi_{A \circ \phi_{j}}\left(t_{i}, t_{i}-\frac{\tau}{d}\right)\left(\psi_{j}\left(t_{i}-\frac{\tau}{d}\right)+\nu L_{0} \Delta_{j-1}\left(t_{i}\right)\right)-\Phi_{A \circ \phi_{j}}\left(t_{i}, t_{i}-\frac{\tau}{d}\right) \nu L_{0} C\left(t_{i}\right) \psi_{j-1}\left(t_{i}-\frac{\tau}{d}\right)$ holds for all $j \in\{2, \ldots, p\}$ and $i \geq r$, so by (7),

$$
\left\{\begin{array}{l}
\dot{\psi}_{j}(t)=A\left(\phi_{j}(t)\right) \psi_{j}(t), \quad \forall t \in J_{i} \\
\psi_{j}\left(t_{i}^{+}\right)=\mathcal{F}_{j}(i) \psi_{j}\left(t_{i}-\frac{\tau}{d}\right)-\Phi_{A \circ \phi_{j}}\left(t_{i}, t_{i}-\frac{\tau}{d}\right) L C\left(t_{i}\right) \psi_{j-1}\left(t_{i}-\frac{\tau}{d}\right)+\Delta_{j-1}^{\sharp}\left(t_{i}\right), i \geq r
\end{array}\right.
$$

for all $j \in\{2, \ldots, p\}$, where $\mathcal{F}_{j}(i)=\Phi_{A \circ \phi_{j}}\left(t_{i}, t_{i}-\tau / d\right)+\nu L_{0} C\left(t_{i}\right)$ and $\Delta_{j-1}^{\sharp}\left(t_{i}\right)=\Phi_{A \circ \phi_{j}}\left(t_{i}, t_{i}-\right.$ $\tau / d) \nu L_{0} \Delta_{j-1}\left(t_{i}\right)$. Integrating the first equality of (8) over $\left(t_{i-1}, t_{i}-\tau / d\right]$ now gives

$$
\left\{\begin{aligned}
\dot{\psi}_{j}(t) & =A\left(\phi_{j}(t)\right) \psi_{j}(t), \quad \forall t \in J_{i} \\
\psi_{j}\left(t_{i}^{+}\right) & =M_{i j} \psi_{j}\left(t_{i-1}^{+}\right)-\Phi_{A \circ \phi_{j}}\left(t_{i}, t_{i}-\frac{\tau}{d}\right) L C\left(t_{i}\right) \psi_{j-1}\left(t_{i}-\frac{\tau}{d}\right)+\Delta_{j-1}^{\sharp}\left(t_{i}\right), i \geq r
\end{aligned}\right.
$$

for all $j \in\{2, \ldots, p\}$, by the definition of the $M_{i j}$ 's from the start of the first part of the proof.

Since the first lines of (4) and (9) (integrated along the $J_{i}$ 's) and the fact that $\tau / d<\nu$ give $\psi_{1}\left(t_{i}-\frac{\tau}{d}\right)=\Phi_{A \circ \phi_{1}}\left(t_{i}-\frac{\tau}{d}, t_{i-1}\right) \psi_{1}\left(t_{i-1}^{+}\right)-\int_{t_{i-1}}^{t_{i}-\tau / d} \Phi_{A \circ \phi_{1}}\left(t_{i}-\frac{\tau}{d}, w\right) \delta_{1}\left(w+\frac{\tau}{d}-r \nu\right) \mathrm{d} w$ and $\psi_{j}\left(t_{i}-\frac{\tau}{d}\right)=\Phi_{A \circ \phi_{j}}\left(t_{i}-\frac{\tau}{d}, t_{i-1}\right) \psi_{j}\left(t_{i-1}^{+}\right)$for all integers $i \geq r$ and $j \in\{2, \ldots, p\}$, we can combine (5) with the second equality of (9) to obtain the discrete time system

$$
\left\{\begin{array}{l}
\psi_{1}\left(t_{i}^{+}\right)=M_{i 1} \psi_{1}\left(t_{i-1}^{+}\right)+\mathcal{N}_{0}(\delta, i) \\
\psi_{j}\left(t_{i}^{+}\right)=M_{i j} \psi_{j}\left(t_{i-1}^{+}\right)+N_{1}(i, j) \psi_{j-1}\left(t_{i-1}^{+}\right)+\mathcal{N}_{1}(\delta, j, i) \text { for all } i \geq r \text { and } j \in\{2, \ldots, p\}
\end{array}\right.
$$

where $N_{1}(i, j)=-\Phi_{A \circ \phi_{j}}\left(t_{i}, t_{i}-\frac{\tau}{d}\right) L C\left(t_{i}\right) \Phi_{A \circ \phi_{j}}\left(t_{i}-\frac{\tau}{d}, t_{i-1}\right)$ and

$$
\mathcal{N}_{1}(\delta, j, i)=\Delta_{1}^{\sharp}\left(t_{i}\right)+\Phi_{A \circ \phi_{j}}\left(t_{i}, t_{i}-\frac{\tau}{d}\right) L C\left(t_{i}\right) \int_{t_{i-1}}^{t_{i}-\tau / d} \Phi_{A \circ \phi_{j}}\left(t_{i}-\frac{\tau}{d}, w\right) \delta_{1}\left(w+\frac{\tau}{d}-r \nu\right) \mathrm{d} w
$$


when $j=2$ and $\mathcal{N}_{1}(\delta, j, i)=0$ if $3 \leq j \leq p$.

Third Part. We first use the function $V(x)=x^{\top} P x$ (where $P=P_{0} / c_{0}$ is from the start of the second part of the proof) to prove that the dynamics for the discrete time variable $\mathcal{D}(i)=$ $\Psi\left(t_{i}^{+}\right)=\left(\psi_{1}\left(t_{i}^{+}\right), \ldots, \psi_{p}\left(t_{i}^{+}\right)\right)$are exponentially ISS with respect to $\delta=\left(\delta_{1}, \delta_{2}\right)$. By substituting in the formula from the second equation in (11), and then recalling that $M_{i j}^{\top} P M_{i j}-P \leq-I$ for all $i$ and $j$ and Young's inequality, and setting $\Delta V(j, i)=V\left(\psi_{j}\left(t_{i}^{+}\right)\right)-V\left(\psi_{j}\left(t_{i-1}^{+}\right)\right)$, we obtain

$$
\begin{aligned}
\Delta V(j, i) \leq & -\psi_{j}^{\top}\left(t_{i-1}^{+}\right) \psi_{j}\left(t_{i-1}^{+}\right)+2\left(M_{i j} \psi_{j}\left(t_{i-1}^{+}\right)\right)^{\top} P\left(N_{1}(i, j) \psi_{j-1}\left(t_{i-1}^{+}\right)+\mathcal{N}_{1}(\delta, j, i)\right) \\
& +\left(N_{1}(i, j) \psi_{j-1}\left(t_{i-1}^{+}\right)+\mathcal{N}_{1}(\delta, j, i)\right)^{\top} P\left(N_{1}(i, j) \psi_{j-1}\left(t_{i-1}^{+}\right)+\mathcal{N}_{1}(\delta, j, i)\right) \\
\leq & -\left|\psi_{j}\left(t_{i-1}^{+}\right)\right|^{2}+\left|\mathcal{N}_{1}(\delta, j, i)\right|^{2}|P|+\left|\psi_{j-1}\left(t_{i-1}^{+}\right)\right|^{2}\left|N_{1}(i, j)\right|^{2}|P| \\
& +\left[2\left\{\left|N_{1}(i, j)\right||P|\left|\mathcal{N}_{1}(\delta, j, i)\right|\right\}\left\{\left|\psi_{j-1}\left(t_{i-1}^{+}\right)\right|\right\}\right. \\
& \left.+2\left(M_{i j} \psi_{j}\left(t_{i-1}^{+}\right)\right)^{\top} P\left(N_{1}(i, j) \psi_{j-1}\left(t_{i-1}^{+}\right)+\mathcal{N}_{1}(\delta, j, i)\right)\right] \\
\leq & -\frac{1}{2}\left|\psi_{j}\left(t_{i-1}^{+}\right)\right|^{2}+N_{2}\left|\psi_{j-1}\left(t_{i-1}^{+}\right)\right|^{2}+\mathcal{N}_{2}(\delta, j, i) \text { for } j=2, \ldots, p \text { and } i \geq r
\end{aligned}
$$

where $N_{2}=\sup \left\{\left|N_{1}(i, j)\right|^{2}|P|+\frac{1}{4}+4\left|M_{i j}\right|^{2}|P|^{2}\left|N_{1}(i, j)\right|^{2}: i \geq 0,1 \leq j \leq p\right\}$ and $\mathcal{N}_{2}(\delta, j, i)=$ $\left|\mathcal{N}_{1}(\delta, j, i)\right|^{2}\left(|P|+4\left|N_{1}(i, j)\right|^{2}|P|^{2}+4\left|M_{i j}\right|^{2}|P|^{2}\right)$, and where the second inequality followed from using the inequality $2 a b \leq \frac{1}{4} a^{2}+4 b^{2}$ three times with appropriate $a$ and $b$ (once with $a=\left|\psi_{j-1}\left(t_{i-1}^{+}\right)\right|$, and then twice using $\left.a=\left|\psi_{j}\left(t_{i-1}^{+}\right)\right|\right)$to upper bound the terms in squared brackets in (12). The sup in the definition of $N_{2}$ is finite because Lemma A.2 implies that $\sup \left\{\left|\Phi_{A \circ \phi_{j}}\left(t_{i}, t_{i}-\tau / d\right)\right|: i \geq 1,1 \leq j \leq p\right\}$ and $\sup \left\{\left|\Phi_{A \circ \phi_{j}}\left(t_{i}-\frac{\tau}{d}, t_{i-1}\right)\right|: i \geq 1,1 \leq\right.$ $j \leq p\}$ are finite and because $C$ is bounded. Also, for each $i \geq r$, we have $\Delta V(1, i) \leq$ $-\psi_{1}^{\top}\left(t_{i-1}^{+}\right) \psi_{1}\left(t_{i-1}^{+}\right)+2\left(M_{i 1} \psi_{1}\left(t_{i-1}^{+}\right)\right)^{\top} P \mathcal{N}_{0}(\delta, i)+\mathcal{N}_{0}(\delta, i)^{\top} P \mathcal{N}_{0}(\delta, i) \leq-\frac{1}{2}\left|\psi_{1}\left(t_{i-1}^{+}\right)\right|^{2}+\mathcal{N}_{3}(\delta, i)$, where $\mathcal{N}_{3}=\left(2 \max _{i}\left|M_{i 1}\right|^{2}|P|^{2}+|P|\right)\left|\mathcal{N}_{0}\right|^{2}$, and using (11) and $2\left(M_{i 1} \psi_{1}\left(t_{i-1}^{+}\right)\right)^{\top} P \mathcal{N}_{0}(\delta, i) \leq$ $\frac{1}{2}\left|\psi_{1}\left(t_{i-1}^{+}\right)\right|^{2}+2\left|M_{i 1}\right|^{2}|P|^{2}\left|\mathcal{N}_{0}(\delta, i)\right|^{2}$. Since the last upper bound on the right side of (12) depends linearly on $\left|\psi_{j-1}\left(t_{i-1}^{+}\right)\right|^{2}$, we can recursively build a strict Lyapunov function for the variable $\mathcal{D}$ to complete the proof; see Appendix A.3 below for complete details.

\section{ILLUSTRATIONS}

Two-Dimensional Example. Consider the special case where

$$
A(t)=\left[\begin{array}{cc}
0 & q+\delta_{A 1}(t) \\
-q+\delta_{A 2}(t) & 0
\end{array}\right], C(t)=\left[\begin{array}{ll}
a+\delta_{C 1}(t) & b+\delta_{C 2}(t)
\end{array}\right]
$$

and $\nu=\frac{1}{q}\left(\frac{\pi}{2}+2 k \pi\right)$, where $a, b$, and $q>0$ are constants and $k$ is any nonnegative integer, for any choice of $B$. We show how we can satisfy Assumption 1 with

$A_{0}=\left[\begin{array}{cc}0 & q \\ -q & 0\end{array}\right], C_{0}=\left[\begin{array}{ll}a & b\end{array}\right], \delta_{A}(t)=\left[\begin{array}{cc}0 & \delta_{A 1}(t) \\ \delta_{A 2}(t) & 0\end{array}\right]$, and $\delta_{C}(t)=\left[\begin{array}{c}\delta_{C 1}(t) \\ \delta_{C 2}(t)\end{array}\right]^{\top}$. 
If we set $\nu L_{0}=\left[\begin{array}{ll}L_{1} & L_{2}\end{array}\right]^{\top}$ for real constants $L_{1}$ and $L_{2}$ to be determined, then

$$
M_{0}(\nu)=\left[\begin{array}{cc}
L_{1} a \sin (q \tau / d)-L_{1} b \cos (q \tau / d) & 1+L_{1} a \cos (q \tau / d)-L_{1} b \sin (q \tau / d) \\
L_{2} a \sin (q \tau / d)-L_{2} b \cos (q \tau / d)-1 & L_{2} a \cos (q \tau / d)-L_{2} b \sin (q \tau / d)
\end{array}\right] .
$$

For $\tau=0$, the eigenvalues $\frac{1}{2}\left(L_{2} a-L_{1} b \pm \sqrt{\left(L_{2} a-L_{1} b\right)^{2}-4\left(1+L_{1} a+L_{2} b\right)}\right)$ of (15) are valued in $(-1,1)$ if $L_{1} a+L_{2} b=-1$ and $L_{1} b-L_{2} a=c$ are satisfied for some $c \in(-1,1)$. For each $c \in(-1,1)$, we can find values $L_{i}$ that satisfy preceding system of equations if $a$ and $b$ are not both zero. Hence, for each $\tau>0$ and $q>0$, we can find a $d>0$ so that $M_{0}(\nu)$ is Schur stable when $a$ and $b$ are not both zero (by choosing $d$ so that $q \tau / d$ is small enough, and using continuity of eigenvalues as functions of the entries of a matrix), which allows us to find the $P_{0}$ required by Assumption 1. For instance, if $q=3.8, a=1, b=0, \tau=1, d=30, \ell=12, L_{1}=-1$, $L_{2}=\frac{1}{2}, \delta_{A 1}=0, \delta_{A 2}=0.04 \sin (t), \delta_{C}=0, k=0$, and $\nu=0.4$, then we can use Mathematica [15] to solve $M_{0}^{\top}(\nu) P_{0} M_{0}(\nu)-P_{0}=-I$ for $P_{0}$ and then use the $\mathcal{G}$ and $\mathcal{H}$ formulas from Remark A.2 (and the fact that $A_{0}^{\top}=-A_{0}$ ) to check that Assumptions 1-2 are satisfied. Hence, for any output delay $r \in \mathbb{N}$, Theorem 1 provides the observer $z_{p}$, where $z_{p}$ is the state of the last subsystem in (2b) and $p=d+\ell r=30+12 r$. Moreover, with $B=\left[\begin{array}{ll}-0.52 & 0\end{array}\right]^{\top}$ and $K=\left[\begin{array}{ll}0.18 & 0\end{array}\right]$, we can use [14] (with $V(t, x)=x^{\top} P_{*} x$ where $P_{*}$ is the solution of $\left(A_{0}^{\sharp}\right)^{\top} P_{*}+P_{*} A_{0}^{\sharp}=-I$ and $A_{0}^{\sharp}=A_{0}+B K, g(t, x)=B, u_{s}(t, x)=K x, f(t, x)=A(t) x, W(x)=0.82|x|^{2}, \mathbf{c}_{1}=0.01$, $\mathbf{c}_{2}=248.11$, and $\left.\mathbf{c}_{3}=0.01\right)$ to check that we can allow any sample rate $\bar{s} \in[0,0.93]$ in the input (in the $g(t)$ as defined in Section II) and establish an ISS result, using part (c) of Theorem 1.

Robotic DC Motor. Consider the case of a single-link direct-drive manipulator actuated by a permanent magnet DC brush motor, which produces this model from [16]:

$$
\begin{aligned}
& \mathcal{M} \ddot{q}+\mathcal{B} \dot{q}+\mathcal{N} \sin (q)=\mathcal{I}, \quad \mathcal{L} \dot{\mathcal{I}}=V_{e}-R \mathcal{I}-K_{B} \dot{q}, \\
& \text { where } \mathcal{M}=\frac{J}{K_{\tau}}+\frac{m \mathcal{L}_{0}^{2}}{3 K_{\tau}}+\frac{\mathcal{M}_{0} \mathcal{L}_{0}^{2}}{K_{\tau}}+\frac{2 \mathcal{M}_{0} R_{0}^{2}}{5 K_{\tau}}, \mathcal{N}=\frac{m \mathcal{L}_{0} G}{2 K_{\tau}}+\frac{\mathcal{M}_{0} \mathcal{L}_{0} G}{K_{\tau}}, \text { and } \mathcal{B}=\frac{B_{0}}{K_{\tau}}
\end{aligned}
$$

and where $B_{0}$ is the viscous friction coefficient at the joint, $G$ is the gravitational constant, $\mathcal{I}(t)$ is the motor armature current, $J$ is the rotor inertia, $K_{B}$ is the back-emf coefficient, the coefficient $K_{\tau}$ characterizes the electromagnetic conversion of armature current to torque, $\mathcal{L}$ is the armature inductance, $\mathcal{L}_{0}$ is the length of the link, $m$ is the mass of the link, $\mathcal{M}_{0}$ is the mass of the load, $q(t)$ is the position of the load (which is the angular motor position), $R_{0}$ is the radius of the load, $R$ is the armature resistance, and $V_{e}$ is the input current voltage. All of the constants in (16) are positive. Our work [17] provided continuous-discrete observers for (16).

However, [17] left open the problem of observer design under input and output delays. Here we help address these remaining challenges for a linearization of (16) around a $C^{1}$ reference 
trajectory $x_{r}(t)=\left(q_{r}(t), \dot{q}_{r}(t), \mathcal{I}_{r}(t)\right)$, which produces the tracking error dynamics

$$
\left\{\begin{array}{l}
\dot{x}_{1}(t)=x_{2}(t), \quad \dot{x}_{2}(t)=b_{1} x_{3}(t)-a_{1} \cos \left(x_{1 r}(t)\right) x_{1}(t)-a_{2} x_{2}(t) \\
\dot{x}_{3}(t)=b_{0} u(g(t)-\tau)-a_{3} x_{2}(t)-a_{4} x_{3}(t), \quad y\left(t_{i}\right)=x_{1}\left(t_{i-r}\right)+\delta_{2}\left(t_{i}\right),
\end{array}\right.
$$

where $x_{1}=q-q_{r}, x_{2}=\dot{q}-\dot{q}_{r}, x_{3}=\mathcal{I}-\mathcal{I}_{r}, u=V_{e}$ is the control, $a_{1}=\mathcal{N} / \mathcal{M}, a_{2}=\mathcal{B} / \mathcal{M}$, $a_{3}=K_{B} / \mathcal{L}, a_{4}=R / \mathcal{L}, b_{0}=1 / \mathcal{L}$, and $b_{1}=1 / \mathcal{M}[17]$. We can now apply our theorem with

$$
A(t)=\left[\begin{array}{ccc}
0 & 1 & 0 \\
-a_{1} \cos \left(x_{1 r}(t)\right) & -a_{2} & b_{1} \\
0 & -a_{3} & -a_{4}
\end{array}\right], A_{0}=\left[\begin{array}{ccc}
0 & 1 & 0 \\
0 & -a_{2} & b_{1} \\
0 & -a_{3} & -a_{4}
\end{array}\right]
$$

and $C(t)=C_{0}=\left[\begin{array}{lll}1 & 0 & 0\end{array}\right]$ and $\delta_{C}=0$, where the only nonzero entry of $\delta_{A}$ is $-a_{1} \cos \left(x_{1 r}(t)\right)$, so $\left|\delta_{*}\right|_{\infty}=\left|\left(\delta_{A}, \delta_{C}\right)\right|_{\infty}=a_{1}$. For instance, using Mathematica [15] to solve $M_{0}^{\top}(\nu) P_{0} M_{0}(\nu)-P_{0}=$ $-I$ for $P_{0}$, we can check that if $a_{2}=b_{1}=a_{3}=0.5, a_{4}=0.1$, and $\tau=1$, then Assumptions 1-2 are satisfied with $\nu=0.3, L_{0}=-(1 / \nu)\left[\begin{array}{lll}0.2 & 0.18 & 0.05\end{array}\right]^{\top}, \ell=9, d=30$, and any $\left|\delta_{*}\right|_{\infty} \in[0,0.0725)$, and then Theorem 1 gives the observer $z_{p}$ where $p=30+9 r$ and $r \in \mathbb{N}$ is the output delay.

\section{CONCLUSIONS}

We provided a new continuous-discrete observer for linear time-varying systems with input delays, uncertainties, sampling in the output observations, and additive uncertainties on the control and outputs. Our work is new even in the special case of LTI systems, and is motivated by many engineering applications where only sampled output values are available. Our sequential observer method produced observers and stabilizing feedbacks without distributed terms. We allow arbitrarily long constant input and output delays, which can differ. We provided sufficient conditions for the sampling rate $\nu$ in the output to satisfy our assumptions, and in future work, we hope to find ways to allow even larger values of $\nu$. We also hope to extend our work to aperiodic sampling in the output, and to use ideas from [1] to cover nonlinear systems.

\section{APPENDICES}

Appendix A.1: Checking Part (ii) from Assumption 1. We prove the following result:

Lemma A.1. Let $\left(A_{0}, C_{0}\right) \in \mathbb{R}^{n_{x} \times n_{x}} \times \mathbb{R}^{n_{y} \times n_{x}}$ be an observable pair, $L_{0}$ be any constant matrix such that $H=A_{0}+L_{0} C_{0}$ is Hurwitz, and the positive values $\tau, d$, and $\nu$ be such that $\tau / d<\nu$. Let the positive definite symmetric matrix $P_{a}$ be such that $P_{a} H+H^{\top} P_{a} \leq-I$. Set

$$
\bar{\gamma}(\nu)=\frac{1}{\nu}\left[\frac{e^{\nu\left|A_{0}\right|}-1-\nu\left|A_{0}\right|}{\nu}+\left|L_{0} C_{0}\right|\left(e^{\nu\left|A_{0}\right|}-1\right)\right]
$$

and $\bar{R}(\nu)=2 \bar{\gamma}(\nu)\left|P_{a}\right|+\left|H^{\top} P_{a} H\right|+2 \nu \bar{\gamma}(\nu)\left|P_{a} H\right|+\nu^{2}\left|P_{a}\right| \bar{\gamma}(\nu)^{2}$. Then if $\nu \bar{R}(\nu) \leq 1 / 2$, then the matrix $P_{0}=(2 / \nu) P_{a}$ satisfies the requirements of part (ii) of Assumption 1. 
Proof. For any $M \in \mathbb{R}^{n_{x} \times n_{x}}$, we have $e^{s M}=I+M \int_{0}^{s} e^{\ell M} \mathrm{~d} \ell$ for all $s \geq 0$ and $e^{M}=$ $I+M+M^{2} \int_{0}^{1} \int_{0}^{m} e^{\ell M} \mathrm{~d} \ell \mathrm{d} m$; the first equality is by the Fundamental Theorem of Calculus, and then the second equality followed by setting $s=1$ and then $s=\ell$ in the first equality. Specializing the second equality to the case where $M=\nu A_{0}$, and specializing the first equality to the case where $M=\left(\nu-\frac{\tau}{d}\right) A_{0}$ and $s=1$, we then have $M_{0}(\nu)=I+\nu H+\nu^{2} \gamma(\nu)$, where

$$
\gamma(\nu)=A_{0}^{2} \int_{0}^{1} \int_{0}^{m} e^{\ell \nu A_{0}} \mathrm{~d} \ell \mathrm{d} m+\left(1-\frac{\tau}{\nu d}\right) L_{0} C_{0} A_{0} \int_{0}^{1} e^{\left(\nu-\frac{\tau}{d}\right) \ell A_{0}} \mathrm{~d} \ell .
$$

This gives $M_{0}(\nu)^{\top} P_{a} M_{0}(\nu)-P_{a}=\nu\left(H^{\top} P_{a}+P_{a} H\right)+\nu^{2}\left(\gamma(\nu)^{\top} P_{a}+P_{a} \gamma(\nu)+H^{\top} P_{a} H\right)+$ $\nu^{3}\left(\gamma(\nu)^{\top} P_{a} H+H^{\top} P_{a} \gamma(\nu)\right)+\nu^{4} \gamma(\nu)^{\top} P_{a} \gamma(\nu)$, by collecting terms. Hence, our choice of $P_{a}$ gives $M_{0}(\nu)^{\top} P_{a} M_{0}(\nu)-P_{a} \leq \nu[-I+\nu R(\nu)]$, where $R(\nu)=\gamma(\nu)^{\top} P_{a}+P_{a} \gamma(\nu)+H^{\top} P_{a} H+$ $\nu\left(\gamma(\nu)^{\top} P_{a} H+H^{\top} P_{a} \gamma(\nu)\right)+\nu^{2} \gamma(\nu)^{\top} P_{a} \gamma(\nu)$, and we can bound and then evaluate the integrals from (A.2) to get $|\gamma(\nu)| \leq \bar{\gamma}(\nu)$ and $|R(\nu)| \leq \bar{R}(\nu)$. Since $\nu \bar{R}(\nu) \leq 1 / 2$, we conclude that $M_{0}(\nu)^{\top} P_{a} M_{0}(\nu)-P_{a} \leq-(\nu / 2) I$, so the lemma follows from our choice of $P_{0}$.

Appendix A.2: Comparison Lemma. We used this lemma in our proof of Theorem 1:

Lemma A.2. Let $\mathcal{A}_{0} \in \mathbb{R}^{n_{x} \times n_{x}}$ and $\delta_{\mathcal{A}}: \mathbb{R} \rightarrow \mathbb{R}^{n_{x} \times n_{x}}$ be piecewise continuous and bounded. Then the fundamental solution $\Phi_{\mathcal{A}}$ associated with $\mathcal{A}(t)=\mathcal{A}_{0}+\delta_{\mathcal{A}}(t)$ satisfies $\left|\Phi_{\mathcal{A}}(t, s)\right| \leq e^{|\mathcal{A}|_{\infty}(t-s)}$ and $\left|\Phi_{\mathcal{A}}(t, s)-e^{\mathcal{A}_{0}(t-s)}\right| \leq e^{(t-s)\left|\mathcal{A}_{0}\right|}\left(e^{(t-s)\left|\delta_{\mathcal{A}}\right|_{\infty}}-1\right)$ for all $s \geq 0$ and $t \geq s$.

Proof. The function $z(t, s)=\Phi_{\mathcal{A}}(t, s)-e^{\mathcal{A}_{0}(t-s)}$ satisfies $\frac{\partial}{\partial t} z(t, s)=\mathcal{A}(t) \Phi_{\mathcal{A}}(t, s)-\mathcal{A}_{0} e^{\mathcal{A}_{0}(t-s)}=$ $\mathcal{A}_{0} z(t, s)+\delta_{\mathcal{A}}(t) \Phi_{\mathcal{A}}(t, s)$ and $z(s, s)=0$ if $t \geq s \geq 0$. Hence, by variation of parameters,

$$
z(t, s)=\int_{s}^{t} e^{\mathcal{A}_{0}(t-r)} \delta_{\mathcal{A}}(r) \Phi_{\mathcal{A}}(r, s) \mathrm{d} r \text { if } t \geq s \geq 0 .
$$

Also, Gronwall's inequality applied to any solution of $\dot{\mu}=\mathcal{A}(t) \mu$ gives $\left|\Phi_{\mathcal{A}}(t, s) \mu(s)\right|=|\mu(t)| \leq$ $e^{|\mathcal{A}|_{\infty}(t-s)}|\mu(s)|$, by the representation $\mu(t)=\mu(s)+\int_{s}^{t} \mathcal{A}(w) \mu(w) \mathrm{d} w$. Since we can choose any $\mu(s) \in \mathbb{R}^{n_{x}}$, this gives $\left|\Phi_{\mathcal{A}}(t, s)\right| \leq e^{|\mathcal{A}|_{\infty}(t-s)}$ (by the definition $\left|\Phi_{\mathcal{A}}(t, s)\right|=\inf \{c \in[0, \infty)$ : $\left|\Phi_{\mathcal{A}}(t, s) x\right| \leq c|x|$ for all $\left.x \in \mathbb{R}^{n_{x}}\right\}$ of the matrix norm of $\Phi_{\mathcal{A}}(t, s)$ ), which we can combine with (A.3) to get $|z(t, s)| \leq \int_{s}^{t} e^{\left|\mathcal{A}_{0}\right|(t-r)} e^{\left(\left|\mathcal{A}_{0}\right|+\left|\delta_{\mathcal{A}}\right|_{\infty}\right)(r-s)} \mathrm{d} r\left|\delta_{\mathcal{A}}\right|_{\infty} \leq e^{(t-s)\left|\mathcal{A}_{0}\right|}\left|\delta_{\mathcal{A}}\right|_{\infty} \int_{s}^{t} e^{\left|\delta_{\mathcal{A}}\right|_{\infty}(r-s)} \mathrm{d} r$, which one integrates to get the second conclusion of the lemma.

Remark A.2. We can sometimes obtain less conservative bounds on $\left|\Phi_{\mathcal{A}}(t, s)\right|$ and $\mid \Phi_{\mathcal{A}}(t, s)$ $e^{\mathcal{A}_{0}(t-s)} \mid$, using special properties of $\mathcal{A}_{0}$. For instance if $\mathcal{A}_{0}^{\top}=-\mathcal{A}_{0}$, then $\left|\Phi_{\mathcal{A}}(t, s)\right| \leq e^{\left|\delta_{\mathcal{A}}\right|(t-s)}$ holds for all $s \geq 0$ and $t \geq s$ (by checking that the time derivative of $V(x)=\frac{1}{2}|x|^{2}$ along all solutions of $\dot{x}=\mathcal{A}(t) x$ satisfies $\dot{V} \leq 2\left|\delta_{\mathcal{A}}\right|_{\infty} V(x(t))$, and then integrating the result), which 
we substitute into (A.3) to get $|z(t, s)| \leq \int_{s}^{t}\left|e^{\mathcal{A}_{0}(t-r)}\right|\left|\delta_{\mathcal{A}}\right|_{\infty} e^{\left|\delta_{\mathcal{A}}\right|_{\infty}(r-s)} \mathrm{d} r=e^{\left|\delta_{\mathcal{A}}\right|_{\infty}(t-s)}-1 .{ }^{1}$ It follows from the first part of our proof of our theorem that the theorem remains true in the $\mathcal{A}_{0}^{\top}=-\mathcal{A}_{0}$ case if we replace $e^{\nu\left|A_{0}\right|}$ in the $\mathcal{G}\left(\delta_{*}\right)$ formula by 1 and replace the formula for $\mathcal{H}$ by $\mathcal{H}\left(\delta_{*}\right)=\left(1+\nu\left|L_{0} C\right|_{\infty}\right) e^{\left|\delta_{A}\right|_{\infty} \nu}+1+\nu\left|L_{0} C_{0}\right|$. We illustrate these points in Section IV.

Appendix A.3: End of Proof of Theorem 1. Applying (12) for $j=p$ and then $j=$ $p-1$, and then adding the results, provides the bound $\Delta V(p, i)+\left(2 N_{2}+1\right) \Delta V(p-1, i) \leq$ $-\frac{1}{2}\left(\left|\psi_{p-1}\left(t_{i-1}^{+}\right)\right|^{2}+\left|\psi_{p}\left(t_{i-1}^{+}\right)\right|^{2}\right)+2\left(N_{2}+1\right) \overline{\mathcal{N}}_{2}(\delta, i)+\left(2 N_{2}+1\right) N_{2}\left|\psi_{p-2}\left(t_{i-1}^{+}\right)\right|^{2}$, where $\overline{\mathcal{N}}_{2}(\delta, i)=$ $\max _{j} \mathcal{N}_{2}(\delta, j, i)$ (which is finite valued because $A$ is bounded), so the $\Delta V(p-1, i)$ cancelled the $N_{2}\left|\psi_{p-1}\left(t_{i-1}^{+}\right)\right|^{2}$ in the $\Delta V(p, i)$ formula. Repeating this process for $\Delta V(p-s, i)$ for larger integers $s \geq 2$ provides constants $N_{3} \geq 0$ and $\omega_{i}>0$ for which this exponentially ISS Lyapunov decay estimate holds along all solutions of (11): $\sum_{j=1}^{p} \omega_{i} \Delta V(j, i) \leq-\omega_{0} \sum_{j=1}^{p} \omega_{i} V\left(\psi_{j}\left(t_{i-1}^{+}\right)\right)+$ $N_{3}|\delta|_{[0, t]}^{2}$. Now the existence of positive constants $c_{a}$ and $c_{b}$ such that the ISS estimate

$$
\left|\Psi\left(t_{i}^{+}\right)\right| \leq c_{a}\left(e^{-c_{b}\left(t_{i}-t_{r}\right)}\left|\Psi\left(t_{r}^{+}\right)\right|+|\delta|_{[0, t]}\right)
$$

holds for all integers $i \geq r$ follows from standard ISS results [18]. Combining (A.4) with the fact that the first equalities in (4) and (9) provide a positive constant $\bar{c}$ such that

$$
|\Psi(t)| \leq \bar{c}\left(\left|\Psi\left(t_{i}^{+}\right)\right|+|\delta|_{[0, t]}\right) \text { for all } t \in J_{i} \text { and } i \geq 0
$$

now allows us to prove part (a) of the theorem. ${ }^{2}$

To prove the remaining conclusions (b)-(c) of the theorem, first note that

$$
z_{p}(t)=x\left(t+\frac{p \tau}{d}-\nu r\right)+\sum_{s=1}^{p} \psi_{s}(t+(p-s) \tau / d)
$$

for all $t \in \mathbb{R}$ (by an induction on $p$ ). Hence, since $\ell \in \mathbb{N}$ satisfies $d \nu=\ell \tau$, and since $p-\ell r=d$, it follows that

$$
z_{p}(t)=x(t+\tau)+\sum_{s=1}^{p} \psi_{s}(t+(p-s) \tau / d)
$$

which we evaluate at $t-\tau$ and combine with (a) to get (b); the $-\tau$ in $e^{-c_{2}(t-\tau)}$ in (3) was used to take the $\frac{(p-s) \tau}{d}-\tau \geq-\tau$ into account. We prove (c) by combining (a) and (A.7), by writing the closed loop $x$ system from (c) as $\dot{x}(t)=A(t) x(t)+B(t) K z_{p}(g(t)-\tau)+\delta_{1}(t)=$

${ }^{1}$ This follows from the orthogonality of the matrix $e^{\mathcal{A}_{0} s}$ for all $s \geq 0$, which follows because $\mathcal{A}_{0}=-\mathcal{A}_{0}^{\top}$ gives $\mathcal{A}_{0} \mathcal{A}_{0}^{\top}=$ $-\mathcal{A}_{0}^{\top} \mathcal{A}_{0}^{\top}=\mathcal{A}_{0}^{\top} \mathcal{A}_{0}$ and so also $I=e^{s \mathcal{A}_{0}^{\top}+s \mathcal{A}_{0}}=e^{s \mathcal{A}_{0}^{\top}} e^{s \mathcal{A}_{0}}$. We also used the relation $x^{\top} \mathcal{A}_{0} x=\frac{1}{2} x^{\top} \mathcal{A}_{0} x+\frac{1}{2} x^{\top} \mathcal{A}_{0}^{\top} x=0$.

${ }^{2}$ Condition (A.5) was used to convert (A.4) into a continuous time decay estimate. To find $\bar{c}$, note that since (4) and (9) give $\left|\dot{\psi}_{j}(t)\right| \leq|A|_{\infty}\left|\psi_{j}(t)\right|+\left|\delta_{1}\right|_{[0, t]}$ for all $t \in J_{i}$, $i$, and $j$, we get $|\dot{\Psi}(t)| \leq \sqrt{2}\left(|A|_{\infty}|\Psi(t)|+\sqrt{p}\left|\delta_{1}\right|_{[0, t]}\right)$, so Gronwall's inequality gives $|\Psi(t)| \leq\left(\nu \sqrt{2 p}\left|\delta_{1}\right|_{[0, t]}+\left|\Psi\left(t_{i}^{+}\right)\right|\right) e^{\sqrt{2} \nu|A|_{\infty}}$ for all $t \in J_{i}$ and $i$, so we can pick $\bar{c}=e^{\sqrt{2} \nu|A|_{\infty}} \max \{\nu \sqrt{2 p}, 1\}$. 
$A(t) x(t)+B(t) K x(g(t))+\delta_{a}(t)$, where $\delta_{a}(t)=B(t) K \sum_{s=1}^{p} \psi_{s}(g(t)-\tau+(p-s) \tau / d)+\delta_{1}(t)$, and then finding $\bar{\gamma} \in \mathcal{K}_{\infty}$ and constants $\bar{c}_{i}>0$ such that $|x(t)| \leq \bar{c}_{1} e^{-\bar{c}_{2} t / 2}|x(t / 2)|+\bar{\gamma}\left(\left|\delta_{a}\right|_{[t / 2, t]}\right)$ for all $t \geq 0$ along solutions of this closed loop system, then using (a), the ISS assumption, and (A.5) and (A.7) to find a constant $c_{*}>0$ and a $\gamma_{*} \in \mathcal{K}_{\infty}$ such that $|x(t / 2)| \leq \gamma_{*}\left(|x(0)|+|\Psi|_{[-\tau, 0]}+|\delta|_{[0, t]}\right)$ and $\left|\delta_{a}\right|_{[t / 2, t]} \leq c_{*}\left(e^{-c_{2} t}|\Psi|_{[-\tau, 0]}+|\delta|_{[0, t]}\right)$ along all solutions of this closed loop system for all $t \geq 0$.

\section{REFERENCES}

[1] M. Nadri, H. Hammouri, and C. Astorga, "Observer design for continuous- discrete time state affine systems up to output injection,” European Journal of Control, vol. 10, no. 3, pp. 252-263, 2004.

[2] F. Mazenc and D. Normand-Cyrot, "Reduction model approach for linear systems with sampled delayed inputs," IEEE Trans. Automat. Control, vol. 58, no. 5, pp. 1263-1268, 2013.

[3] A. Gonzalez, P. Garcia, P. Albertos, P. Castillo, and R. Lozano, "Robustness of a discrete-time predictor-based controller for time-varying measurement delay," Control Engineering Practice, vol. 20, no. 2, pp. 102-110, 2012.

[4] N. Kazantzis, K. Chong, and A. Parlos, "Control-relevant discretization of nonlinear systems with time-delay using TaylorLie series," ASME Journal of Dynamic Systems, Measurement, and Control, vol. 127, no. 1, pp. 153-159, 2005.

[5] A. Jazwinski, Stochastic Processes and Filtering Theory. Mineola, NY: Dover Publications, 1970.

[6] F. Deza, E. Busvelle, J.-P. Gauthier, and D. Rakotopara, "High gain estimation for nonlinear systems," Systems and Control Letters, vol. 18, no. 4, pp. 259-299, 1992.

[7] G. Goffaux, A. Vande Wouwer, and O. Bernard, "Improving continuous- discrete interval observers with application to microalgae-based bioprocess," Journal of Process Control, vol. 19, no. 7, pp. 1182-1190, 2009.

[8] F. Mazenc and E. Fridman, "Predictor-based sampled-data exponential stabilization through continuous-discrete observers," Automatica, vol. 63, pp. 74-81, 2016.

[9] I. Karafyllis and M. Krstic, "Stabilization of nonlinear delay systems using approximate predictors and high-gain observers," Automatica, vol. 49, pp. 3623-3631, 2013.

[10] M. Najafi, S. Hosseinnia, F. Sheikholeslam, and M. Karimadin, "Closed-loop control of dead time systems via sequential sub-predictors," International Journal of Control, vol. 86, no. 4, pp. 599-609, 2013.

[11] F. Mazenc and M. Malisoff, "Stabilization and robustness analysis for time-varying systems with time-varying delays using a sequential subpredictors approach," Automatica, vol. 82, pp. 118-127, 2017.

[12] F. Mazenc and M. Malisoff, "Continuous-discrete sequential observers under sampling and input delays," in Proceedings of the American Control Conference, Milwaukee, WI, 2018, pp. 5156-5160.

[13] H. Khalil, Nonlinear Systems, Third Edition. Upper Saddle River, NJ: Prentice Hall, 2002.

[14] F. Mazenc, M. Malisoff, and T. Dinh, "Robustness of nonlinear systems with respect to delay and sampling of the controls," Automatica, vol. 49, no. 6, pp. 1925-1931, 2013.

[15] Mathematica, "The world's definitive system for modern technical computing," 2015, www.wolfram.com/mathematica/.

[16] D. Dawson, J. Carroll, and M. Schneider, "Integrator backstepping control of a brush DC motor turning a robotic load," IEEE Trans. Control Systems Technology, vol. 2, no. 3, pp. 233-244, 1994.

[17] F. Mazenc, V. Andrieu, and M. Malisoff, "Design of continuous-discrete observers for time-varying nonlinear systems," Automatica, vol. 57, no. 7, pp. 135-144, 2015.

[18] Z.-P. Jiang and Y. Wang, "Input-to-state stability for discrete-time nonlinear systems," Automatica, vol. 37, pp. 857-869, 2001. 